\title{
KAPILLAR-GASCHROMATOGRAPHISCHE ANALYSE VON APFEL-AROMASTOFFEN
}

In den letzten Jahrzehnten durchgeführte Untersuchungen beweisen, daß die Fruchtaromen aus einer großen Zahl flüchtiger Verbindungen bestehen, die nur in geringen Mengen vorliegen.

Die qualitative, besonders aber quantitative Zusammensetzung der Aromastoffe hängt stark von Sorte, Standort, Vegetationsablauf, Lagerbedingungen usw. ab und verändert sich stetig während des Wachstums, der Reifung und Lagerung $\left[{ }^{1-3}\right]$.

In der Estnischen SSR sind bis jetzt keine Untersuchungen auf diesem Gebiet durchgeführt, deshalb wurden hier zum ersten Mal Ergebnisse über Aromastoffe örtlicher Apfel veröffentlicht.

Es wurden Apfel der Sorten Antonowka, Wealthy (Tartuer Rosenapfel) und von Dr. A. Siimon Nr. 308 untersucht, die von der Obstanlage Polli des Estnischen Wissenschaftlichen Instituts für Ackerbau und Meliorationswesen stammten (Ernte des Jahres 1971).

Um die Veränderung der Zusammensetzung der Aromastoffe je nach dem Reifezustand zu verfolgen, wurden auch unreife, am 5. August 1972 geerntete Apfel der Sorte Antonowka verwendet.*

Für die Anreicherung wurde $800 \mathrm{ml}$ Apfelsaft mit Diäthyläther extrahiert und der Extrakt vorsichtig auf $0,5 \mathrm{ml}$ eingeengt.

Die gaschromatographischen Untersuchungen wurden mit einem Gerät «Chrom 31» («Laboratorni Přistroje», Praha) unter Verwendung des Flammenionisationsdetektors durchgeführt.

Für die Trennung des Aromakonzentrates wurde eine mit Squalan (May \& Baker Ltd., Dagenham, England) beschichtete Kapillartrennsäule (50 m lang, innerer Durchmesser $0,25 \mathrm{~mm}$ ) benutzt. Als Trägergas diente Helium, dessen Druck vor der Trennsäule $1,68-1,72 \mathrm{~kg} / \mathrm{cm}^{2}$ war.

Von den Apfelaromakonzentraten wurden $5 \mu \mathrm{l}$ mit einer $10 \mu \mathrm{l}$ Hamilton-Spritze in den Gas-Chromatograph injiziert und die Trennung unter isothermischen Bedingungen bei Temperaturen $60^{\circ}$ und $100^{\circ} \mathrm{C}$ durchgeführt.

Die getrennten Peaks wurden durch Retentionsindexe identifiziert. Es wurden Retentionsindexe unter denselben Bedingungen, wie oben beschrieben, für verschiedene Ester, Alkohole und Carbonylverbindungen bestimmt, die zusammen mit Literaturangaben $\left[{ }^{4,5}\right.$ ] in Tab. 1 zusammen-

* Wissenschaftlicher Mitarbeiterin Eevi Jaama (Estnisches Wissenschaftliches Institut für Ackerbau und Meliorationswesen, Polli) wird gedankt für das Versuchsmaterial. 
हี

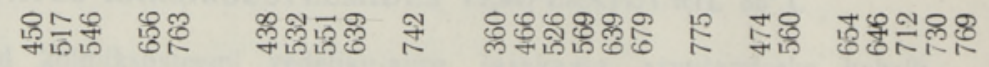

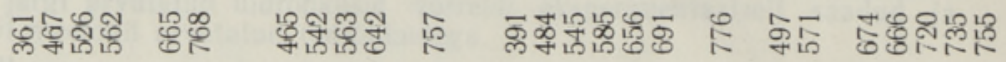

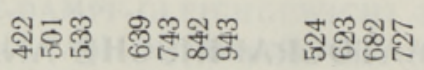

움

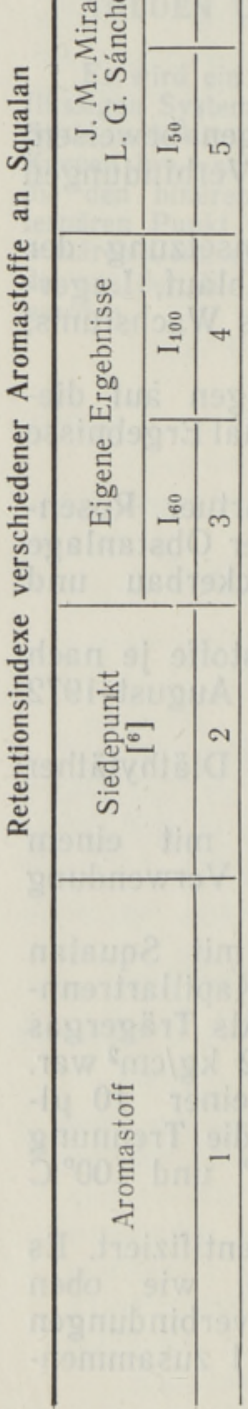

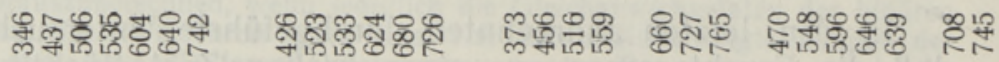

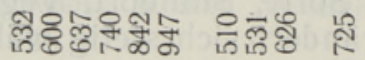

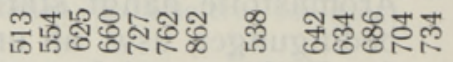

ஜֶ:

เ

송ㅎㅀ융

굼 考 迠

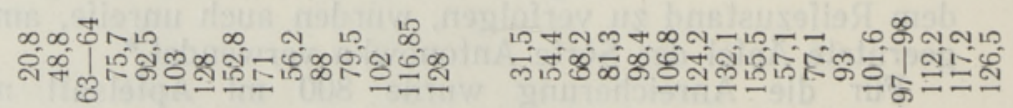

范

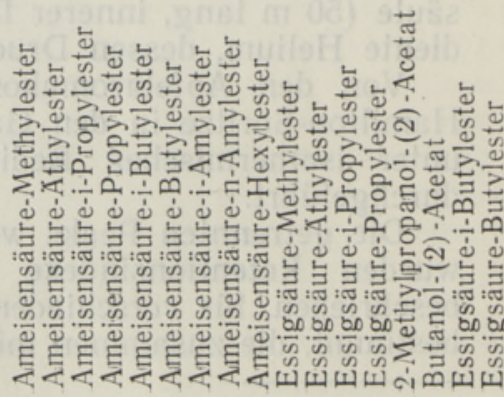




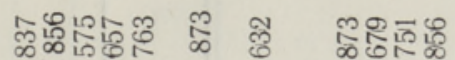

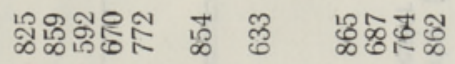

왕

䓵莡

范

高

吕

ซ్ํำ สำ

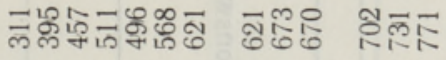

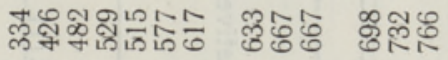

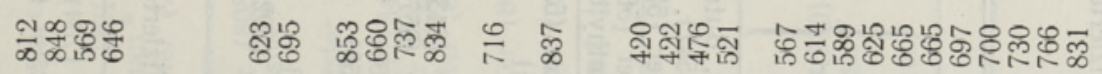

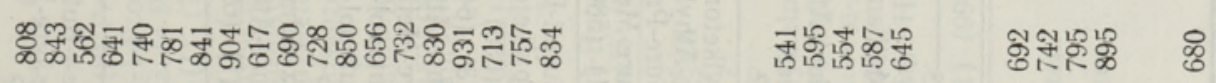
동용
형 홍

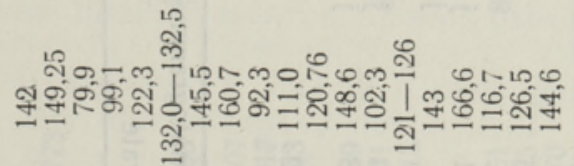

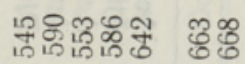

$\mathscr{\wp}$

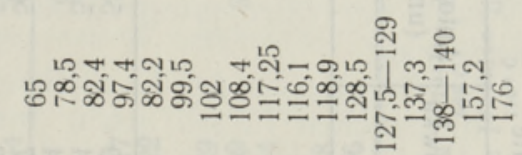

以的的的

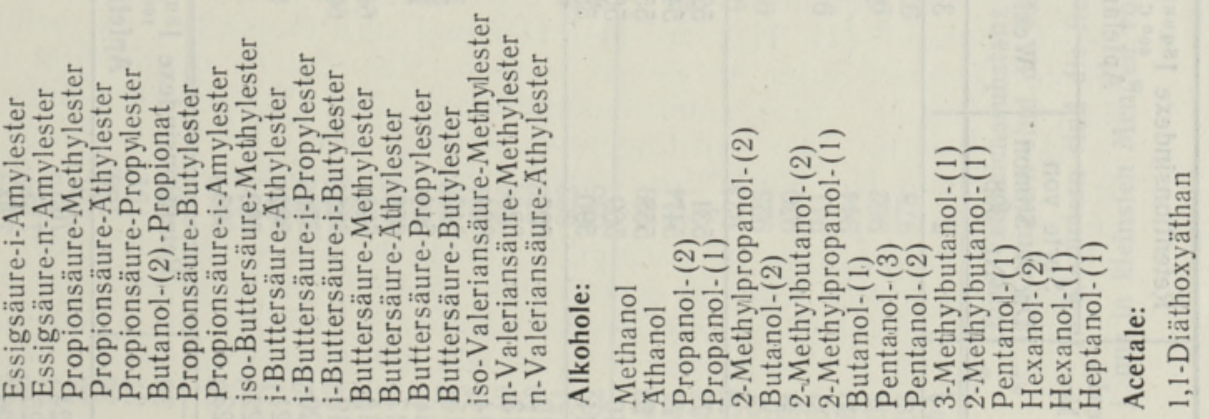


$\frac{1}{\text { a }}$

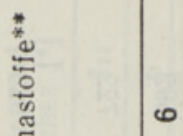

|

范

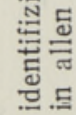

苕

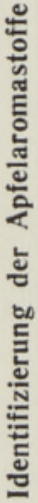
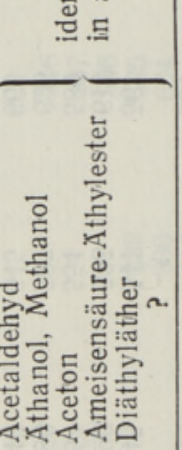

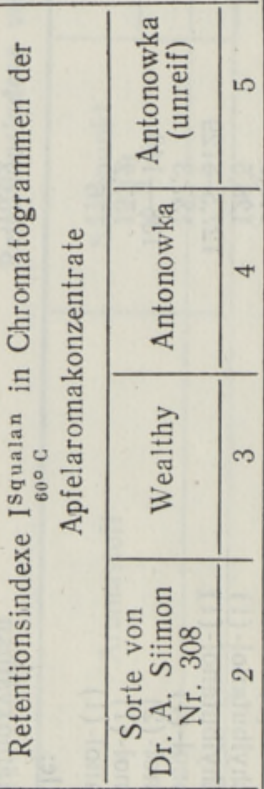

竎芹

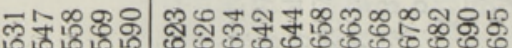

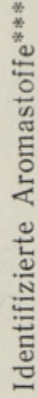

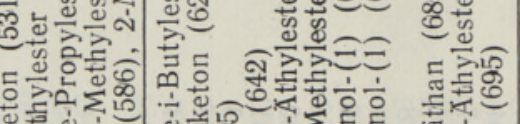

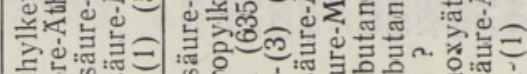

牙零:

戀

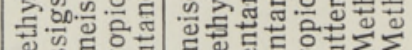

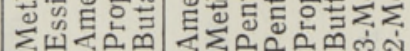

을

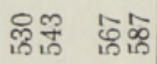

내능요

กิ่ สำ ชึ \%

ถิำเำำ

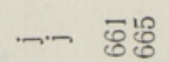

운

亲

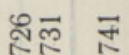

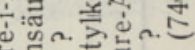

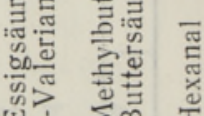

w?

iิ

s

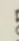

突

政

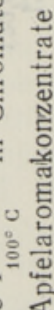

女

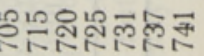

ลัละัฒลัล็ำ 


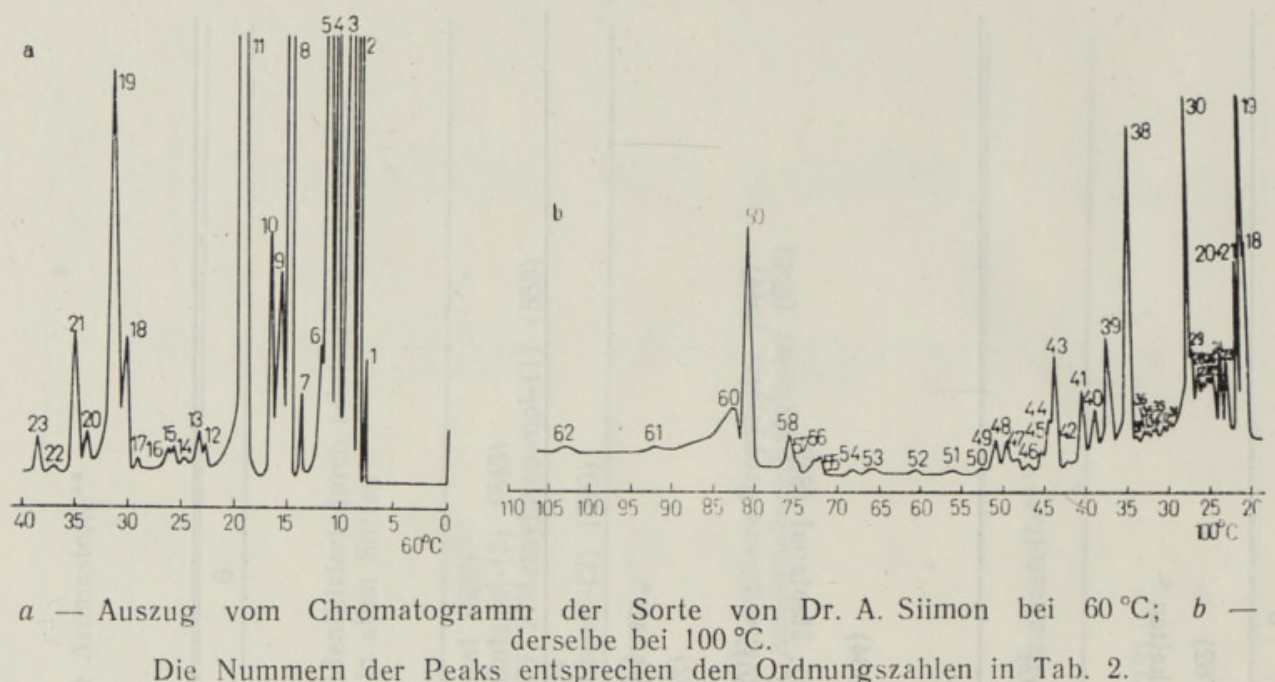

gefaßt sind. Bei Estern, Aldehyden und Ketonen ergibt es ganz gute Übereinstimmung mit den Daten von J. M. Mira und L. G. Sánchez [ ${ }^{4}$; bei Alkoholen dagegen sind unsere Ergebnisse um 20 bis 40 Einheiten kleiner.

Die O. W. McReynolds-Daten unterscheiden sich bei allen Verbindungen um 13 bis 39 Einheiten. Auch hier kann man bei Alkoholen die größte Differenz bemerken.

In beiden Fällen wurde mit gefüllten Trennsäulen gearbeitet, deshalb kann der Einfluß des verwendeten Trägermaterials einer der Gründe des Unterschiedes sein.

Die gaschromatographische Trennung bei $60^{\circ} \mathrm{C}$ ist für Komponenten, deren Retentionsindexe zwischen 500 und 700 liegen, und bei $100^{\circ} \mathrm{C}$ für Substanzen mit Retentionsindexen von 700 bis 900 geeignet. Die ersten 6 Peaks der Chromatogramme wurden durch relative Retentionswerte identifiziert.

Die Ergebnisse der Identifizierung werden in Tab. 2 zusammengefaßt.

In manchen Fällen kann man auch ohne Vergleichssubstanz, von Gesetzmäßigkeiten in Retentionsindexen ausgehend voraussetzen, was für ein Aromastoff einem der Peaks entspricht. (In Tab. 2 mit * bezeichnet).

Wie aus Tab. 2 ersichtlich, sind viele von den Peaks bei allen Sorten vorhanden. Da die Enzyme nicht inhibiert wurden, erscheinen als Hauptkomponenten Butanol-(1), 2-Methylbutanol-(2), 3-Methylbutanol-(1), 2-Methylbutanol-(1), Hexanol-(1), Hexanal und Heptanal, die beim Zerfall des Zellverbandes entstehen können. Von Estern dominieren Essigsäure-Athylester, Essigsäure-Isoamylester und Buttersäure-Butylester.

Als Resultat der vorliegenden Arbeit sind bei der Sorte von Dr. A. Siimon Nr. 30860 , in Wealthy 37, in Antonowka (reif) 45 Verbindungen identifiziert worden. Die unreifen Apfel der Sorte Antonowka enthalten Aromastoffe in geringeren Mengen (29 Verbindungen).

Die verwendete Kapillar-gaschromatographische Untersuchung ist für die Trennung solcher komplizierter Gemische, wie es die Fruchtaromakonzentrate sind, viel besser geeignet als die gepackten Säulen [7]. 


\section{I T E R A T UR}

1. Drawert F., Heima nn W., Emberger R., Tress l R., Chromatographia, 2, 57 (1969).

2. Drawert F., Heimann W., Emberger R., Tress 1 R., Phytochemistry, 7, 881 (1968).

3. Drawert F., Heimann W., Emberger R., Tress 1 R., Z. Lebensmitt. Untersuch. - Forsch., 140, 2. Heft, 65 (1969).

4. M i r a J. Martin, S á n c he z L. Gascó, Anal. Chim. Acta, 50, 315 (1970).

5. McReynolds W. O., Gas Chromatographic Retention Data, Preston Technical Abstracts Company, 1966.

6. Handbook of Chemistry and Physics, 51st Edition, The Chemical Rubber CO. Cleveland, Ohio, 1970-1971.

7. Л и пи с Б. В., С околов а А. Ф., М а м а ко в а Я. А., В кн.: Новые методы технол. и контроля консервн. и винодельн. произ-ва, Кишинев, 1972, с. 111.

Institut für Kybernetik

der Akademie der Wissenschaften der Estnischen SSR
Eingegangen am 10. Jan. 1974

\section{Malle EISEN}

\section{OUNA AROOMIAINETE KAPILLAAR-GAASIKROMATOGRAAFILISE ANALUUUSI TULEMUSED}

Kapillaar-gaasikromatograafia abil uuriti Eesti NSV-s kasvatatud kolme õunasordi - Dr. A. Siimoni seemik nr. 308, 'Antonovka' (koristusküpsed ja toored viljad) ja 'Wealthy' ('Tartu roosōun') — aroomiaineid. Retentsiooniindeksite alusel identifitseeriti kuni 60 individuaalset ühendit.

\section{Малле ЭПЗЕН}

\section{КАПИЛЛЯРНО-ГАЗОХРОМАТОГРАФИЧЕСКОЕ ОПРЕДЕЛЕНИЕ АРОМАТИЧЕСКИХ ВЕЩЕСТВ ЯБЛОК}

Капиллярно-газохроматографически изучали летучие органические вещества, придающие вкус и аромат яблокам трех сортов - сеянец др. А. Сиимона № 308, 'Анто новка' (зрелые и незрелые плоды) и 'Wealthy' ('Плодородное'). При помощи индексов удерживания идентифицировано до 60 индивидуальных соединений. 